\title{
Real-Time Kinematic Positioning for Unmanned Air Vehicles
}

\author{
Patrick Henkel \\ Technische Universität München \\ Theresienstrasse 90 \\ 80333 München \\ +49-89-289-23462 \\ patrick.henkel@tum.de
}

\author{
Andreas Sperl \\ Advanced Navigation Solutions - ANavS GmbH \\ Heßstrasse 36 \\ 80397 München, Germany \\ +49-89-89056721 \\ andreas.sperl@anavs.de
}

\begin{abstract}
Real-Time Kinematic (RTK) positioning with lowcost mass-market Global Navigation Satellite System (GNSS) receivers and antennas is attractive for precise landing of Unmanned Air Vehicles (UAV). Code multipath mitigation and a reliable resolution of the carrier phase integer ambiguities are two challenges.

In this paper, we describe an RTK method which estimates a code multipath parameter for each double difference to fully exploit the temporal correlation of multipath and to prevent a mapping of the multipath into the baseline and ambiguities. The ambiguity fixing is performed in two phases: In the first phase, sets of integer candidate vectors are derived from the float solution at multiple epochs. We merge the sets of candidate vectors to increase the likelihood of including the correct candidate vector in the set of candidates. In the second phase, we track each candidate vector by determining a single epoch baseline estimate for each candidate vector. The respective measurement residuals are accumulated over time to increase the likelihood of selecting the correct candidate.
\end{abstract}

The proposed method was applied to real measurements from two low-cost ANavS GPS modules and enabled a millimeterlevel positioning accuracy despite code multipath of up to $50 \mathrm{~m}$.

\section{Table of Contents}

1. INTRODUCTION...$\ldots \ldots \ldots \ldots \ldots \ldots \ldots \ldots \ldots$

2. Measurement and State Space Models ...... 1

3. RTK POSITIONING

WITH MULTIPATH ESTIMATION ...............

4. Measurement Results ..................... 5

5. Conclusion ..............................6 6

REFERENCES .............................6

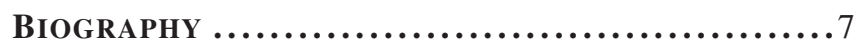

\section{INTRODUCTION}

The precise relative positioning of a kinematic GNSS receiver with respect to a reference receiver using carrier phase measurements and integer ambiguity resolution is called RTK positioning. Double differences are performed to eliminate clock errors, receiver/ satellite biases, and to reduce orbital errors and atmospheric delays. If the distance to the closest reference station exceeds $10 \mathrm{~km}$, reference measurements can be obtained from a virtual reference station to eliminate ionospheric delays and orbital errors more accurately as described by Landau et al. in [1] and by Vollath et al. in [2].

Today, there are two challenges for RTK positioning: the

978-1-4673-7676-1/16/\$31.00 (c)2016 IEEE estimation/ suppression of code multipath and the reliable resolution of the carrier phase integer ambiguities.

Code multipath can be of several tens of metres and, thus, clearly exceeds the wavelength of the carrier signal. Consequently, code multipath is very critical for ambiguity resolution. Choke ring antennas can suppress the multipath but can hardly be used in numerous applications due to restrictions on the size, weight and price of the antennas. Sidereal filtering exploits the repeatability of the satellite constellation, i.e. the multipath is estimated by averaging the code residuals over epochs with equal satellite constellation. This sidereal filtering requires measurements of several days and can only be applied for stationary receivers. It is not applicable for kinematic positioning. Therefore, we estimate an additional code multipath parameter for each double difference to exploit the temporal correlation of code multipath and to prevent a mapping of the code multipath into the baseline and ambiguities.

The Least-Squares Ambiguity Decorrelation Adjustment (LAMBDA) method of Teunissen [3] is widely used for carrier phase ambiguity resolution. It includes an integer decorrelation transformation and a sequential tree search to find the integer candidate vector that is closest to the float solution. There are two drawbacks of the performed minimization of the sum of squared ambiguity residuals in the LAMBDA method: First, any integer error in the float solution remains in the fixed solution and can not be recognized from the ambiguity residuals. Secondly, the method fully relies on the correctness of the covariance matrix. Any unmodeled temporal correlations of the measurements (e.g. due to phase multipath and/ or code multipath) might lead to a biased covariance matrix. A biased covariance matrix means a distorted search space. Consequently, the correct candidate vector might be no longer included in the search space.

We describe an extended fixing method, which determines sets of integer vectors at multiple epochs to increase the likelihood of including the correct integer vector in the set of candidate vectors. Additionally, a fixed baseline solution is determined for each candidate vector and the residuals are accumulated over time. The candidate with a stable baseline and a small accumulated sum of squared residuals is selected to increase the likelihood of correct ambiguity fixing.

\section{Measurement and State Space MODELS}

In this section, we introduce our models for the double difference (DD) carrier phase and pseudorange measurements as well as the considered state space models. We use the following notation: 
Eq. (4) as:

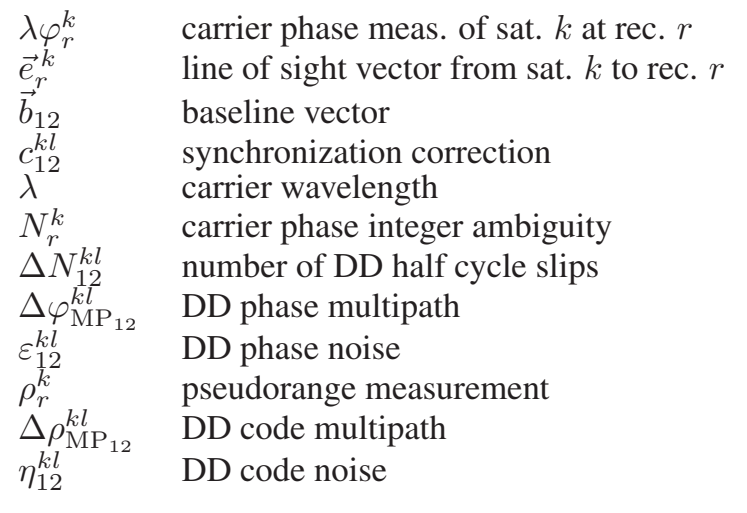

The double difference (DD) carrier phase measurement of two low-cost single frequency GNSS receivers is modeled for a short baseline as

$$
\begin{aligned}
\lambda \varphi_{12}^{k l}= & \vec{e}_{1}^{k l} \vec{b}_{12}+c_{12}^{k l}+\lambda N_{12}^{k l}+\frac{\lambda}{2} \Delta N_{12}^{k l} \\
& +\lambda \Delta \varphi_{\mathrm{MP}_{12}}^{k l}+\varepsilon_{12}^{k l},
\end{aligned}
$$

with the synchronization correction [6] [7]

$$
\begin{aligned}
c_{12}^{k l}= & \left(\vec{e}_{1}^{k}\left(\vec{x}_{2}-\vec{x}_{1}^{k}\right)-\vec{e}_{1}^{l}\left(\vec{x}_{2}-\vec{x}_{1}^{l}\right)\right) \\
& -\left(\vec{e}_{2}^{k}\left(\vec{x}_{2}-\vec{x}_{2}^{k}\right)-\vec{e}_{2}^{l}\left(\vec{x}_{2}-\vec{x}_{2}^{l}\right)\right) .
\end{aligned}
$$

Similarly, the DD pseudorange measurements are modeled as

$$
\rho_{12}^{k l}=\vec{e}_{1}^{k l} \vec{b}_{12}+c_{12}^{k l}+\Delta \rho_{\mathrm{MP}_{12}}^{k l}+\eta_{12}^{k l}
$$

The synchronization correction can be determined with submillimetre level accuracy as shown in [7], which leaves the baseline vector $\vec{b}_{12}$, the DD integer ambiguities $N_{12}^{k l}$, the DD code multipath $\Delta \rho_{\mathrm{MP}_{12}}^{k l}$ and the DD phase multipath $\Delta \varphi_{12}^{k l}$ as unknowns. The temporal behaviour of these state parameters is described by the state space model.

We model the pseudorange and phase multipath as scaled Random-walk processes, i.e.

$$
\begin{aligned}
\Delta \rho_{\mathrm{MP}_{r, n}}^{k} & =\alpha_{n} \Delta \rho_{\mathrm{MP}_{r, n-1}}^{k}+\left(1-\alpha_{n}\right) \eta_{\Delta \rho_{\mathrm{MP}_{r, n}}^{k}} \\
\lambda \Delta \varphi_{\mathrm{MP}_{r, n}}^{k} & =\beta_{n} \lambda \Delta \varphi_{\mathrm{MP}_{r, n-1}}^{k}+\left(1-\beta_{n}\right) \eta_{\lambda \Delta \varphi_{\mathrm{MP}_{r, n}}^{k}}
\end{aligned}
$$

where the scaling factors $\alpha_{n} \in[0,1]$ and $\beta_{n} \in[0,1]$ were introduced for the following reasons:

- modeling of both static multipath ( $\alpha_{n}$ equal to 1 results in deterministic multipath) and kinematic multipath ( $\alpha_{n}$ equal to 0 corresponds to white noise)

- variance of multipath is bounded for $n \rightarrow \infty$ if $\alpha_{n}<1$

Consequently, the parameters $\alpha_{n}$ and $\beta_{n}$ should be a function of the speed of the object, i.e. the values should be reduced for increasing speed.

The code multipath at epoch $n$ can be further developed with

$$
\begin{aligned}
\Delta \rho_{\mathrm{MP}_{r, n}}^{k}= & \left(\prod_{i=1}^{n} \alpha_{i}\right) \Delta \rho_{\mathrm{MP}_{r, 0}}^{k} \\
& +\sum_{i=0}^{n-1}\left(\prod_{j=n-i+1}^{n} \alpha_{j}\right)\left(1-\alpha_{n-i}\right) \eta_{\Delta \rho_{\mathrm{MP}_{r, n-i}}^{k}}
\end{aligned}
$$

The phase multipath can be developed in a similar manner. We limit our further analysis to the code multipath, as the application of the methodology to the phase multipath is straightforward. We model the change of the multipath over time as white Gaussian noise, i.e.

$$
\eta_{\Delta \rho_{\mathrm{MP}_{r, n}}^{k}} \sim \mathcal{N}\left(0, \sigma_{\Delta \rho_{\mathrm{MP} r, n}^{k}}^{2}\right)
$$

Thus, the variance of the code multipath follows as

$$
\begin{aligned}
\sigma_{\Delta \rho_{\mathrm{MP} r, n}^{k}}^{2}= & \left(\prod_{i=1}^{n} \alpha_{i}^{2}\right) \sigma_{\Delta \rho_{\mathrm{MP}_{r, 0}}^{2}}^{2} \\
& +\sum_{i=0}^{n-1}\left(\prod_{j=n-i+1}^{n} \alpha_{j}^{2}\right)\left(1-\alpha_{n-i}\right)^{2} \sigma_{\eta_{\Delta \rho_{\mathrm{MP}}^{k}, n-i}^{2}} .
\end{aligned}
$$

The variances can be further simplified in case of a constant $\alpha_{n}$ and constant $\sigma_{\eta_{\Delta \rho_{\mathrm{MP}}^{k}, n-i}^{2}}^{2}$. We use a finite geometric series expansion to simplify Eq. (7) to

$$
\sigma_{\Delta \rho_{\mathrm{MP}_{r, n}}^{k}}^{2}=\alpha^{2 n} \sigma_{\Delta \rho_{\mathrm{MP}_{r, 0}}^{k}}^{2}+(1-\alpha)^{2} \frac{1-\alpha^{2 n}}{1-\alpha^{2}} \sigma_{\Delta \rho_{\mathrm{MP}_{r, n}}^{k}}^{2} .
$$

For large $n$, the variances converge to the finite value

$$
\lim _{n \rightarrow \infty} \sigma_{\Delta \rho_{\mathrm{MP}_{r, n}}^{k}}^{2}=\frac{(1-\alpha)^{2}}{1-\alpha^{2}} \sigma_{\Delta \rho_{\mathrm{MP}_{r}}^{k}}^{2} .
$$

The baseline is integrated over $\delta t$ using the velocity $\dot{\vec{b}}_{12}$ and the integer ambiguities are constant, which results in the linear state space model

$$
\begin{aligned}
& x_{n}=\left(\begin{array}{c}
\vec{b}_{12} \\
\dot{\vec{b}}_{12} \\
N_{12} \\
\Delta \rho_{\mathrm{MP}_{12}} \\
\lambda \Delta \varphi_{\mathrm{MP}_{12}}
\end{array}\right)_{n} \\
& =\left(\begin{array}{ccccc}
I & \delta t \cdot I & & & \\
& I & & & \\
& & I & & \\
& & & \alpha \cdot I & \\
& & & & \beta \cdot I
\end{array}\right)\left(\begin{array}{c}
\vec{b}_{12} \\
\dot{\vec{b}}_{12} \\
N_{12} \\
\Delta \rho_{\mathrm{MP}_{12}} \\
\lambda \Delta \varphi_{\mathrm{MP}_{12}}
\end{array}\right)_{n-1} \\
& +\eta_{x_{n}},
\end{aligned}
$$

with the process noise $\eta_{x_{n}} \sim \mathcal{N}\left(0, \Sigma_{\eta_{x_{n}}}\right)$. 


\section{RTK POSITIONING WiTH MULTIPATH ESTIMATION}

In this section, the RTK positioning with multipath estimation is described. After a short introduction, we focus on our contributions and their advantages over the state of the art.

The measurement vector at epoch $n$ includes the synchronized and cycle slip corrected DD carrier phase and pseudorange measurements and stochastic a priori information $\bar{x}$ on the state parameters at time $t_{n}$, i.e.

$$
z_{n}=\left(\begin{array}{c}
\lambda \varphi_{12}\left(t_{n}\right)-c_{12}\left(t_{n}\right)-\lambda / 2 \Delta \check{N}_{12}\left(t_{n}\right) \\
\rho_{12}\left(t_{n}\right)-c_{12}\left(t_{n}\right) \\
\bar{x}\left(t_{n}\right)
\end{array}\right)
$$

where $\lambda \varphi_{12}, \rho_{12}, c_{12}$ and $\check{N}_{12}$ are $(K-1) \times 1$ vectors for $K$ visible satellites. The measurements can be expressed as linear functions of the state vector $x_{n}$, which is given by

$$
x_{n}=\left(\begin{array}{c}
\vec{b}_{12}\left(t_{n}\right) \\
N_{12}\left(t_{n}\right) \\
\Delta \rho_{\mathrm{MP}_{12}}\left(t_{n}\right)
\end{array}\right) \text {. }
$$

Obviously, the system of measurement equations is illconditioned. However, the estimation of $x_{n}$ can be substantially improved if a state space model is used. For static receivers, the state transition model is very strong, i.e.

$$
x_{n}=\Phi x_{n-1}+\eta_{x_{n}} \quad \text { with } \quad \Phi=1 .
$$

The covariance matrix of the process noise $\eta_{x_{n}} \sim \mathcal{N}\left(0, \Sigma_{x_{n}}\right)$ describes the change of the DD code multipath between two subsequent epochs, i.e.

$$
\Sigma_{x_{n}}=\left(\begin{array}{ccc}
0 & 0 & 0 \\
0 & 0 & 0 \\
0 & 0 & \Sigma_{\Delta \rho_{\mathrm{MP}_{12}}}
\end{array}\right)
$$

A Kalman filter [4] is used to estimate the state parameters. It includes the state prediction

$$
\begin{aligned}
\hat{x}_{n}^{-} & =\Phi \hat{x}_{n-1}^{+} \\
\Sigma_{\hat{x}_{n}^{-}} & =\Phi \Sigma_{\hat{x}_{n-1}^{+}} \Phi^{\mathrm{T}}+\Sigma_{x_{n}},
\end{aligned}
$$

and the state update

$$
\begin{aligned}
\hat{x}_{n}^{+} & =\hat{x}_{n}^{-}+K_{n}\left(z_{n}-H_{n} \hat{x}_{n}^{-}\right) \\
\Sigma_{\hat{x}_{n}^{+}} & =\left(1-K_{n} H_{n}\right) \Sigma_{\hat{x}_{n}^{-}} .
\end{aligned}
$$

\section{- Float solution:}

In the state of the art RTK positioning [1], the 3D baseline and DD ambiguities are estimated from DD phase and code measurements. The code multipath is not considered as a parameter to be estimated. It is implicitly assumed that it can be mapped to the code noise, and the respective variance is increased accordingly.

Unfortunately, this assumption is not valid as the code multipath is having a substantial deterministic part (especially for static situations), which can not be reduced by averaging over multiple epochs and results in a significant temporal correlation. If the code multipath exceeds the assumed code noise variance, a certain part of the multipath is also mapped to the ambiguities and baseline estimates, which results in a heavily biased float solution.

However, the temporal correlation of multipath could be fully exploited in a state space model. We include a multipath parameter for each DD in the float solution to obtain the following advantages:

- prevent mapping of multipath errors to ambiguities and baseline

- exploit temporal correlation of multipath

- better use of code measurements by setting code noise variance to variance of pure noise

- Triggering of fixing:

The well-known LAMBDA method of Teunissen [3] uses the float ambiguity covariance matrix for ambiguity fixing. The covariance matrix of the float ambiguity solution might be erroneous for the following reasons:

- covariance matrix of DD phase and code measurements is not accurately known, i.e. the correlation between the DD measurements is not properly modeled or estimated

- the phase multipath and its temporal correlation are not taken into account

- receiver tracking errors are not accurately considered in the measurement model

As these errors often lead to an underestimation of the actual uncertainty, we use the stability of the float solution as additional criterion to trigger the fixing. The stability is determined from the maximum variation of the float baseline estimate $\vec{b}_{12}^{(i)}$, whereas the maximization is taken over the last $j_{\mathrm{s}}=\left\{0, \ldots, j_{\max }\right\}$ epochs and over the three coordinates $i \in\{1,2,3\}$, i.e.

$$
\max _{i \in\{1,2,3\}}\left(\max _{j \in j_{\mathrm{s}}} \hat{\vec{b}}_{12}^{(i)}\left(t_{n-j}\right)-\min _{j \in j_{\mathrm{s}}} \hat{\vec{b}}_{12}^{(i)}\left(t_{n-j}\right)\right) .
$$

A stable float solution reduces the likelihood of large errors in the float solution, which is a major advantage over any covariance-only based triggering.

- Candidate search:

The LAMBDA method [3] determines integer candidates in a sequential tree search. In the state of the art approach of [3], the candidates are determined at a certain epoch using the respective float solution, which has some-how converged. The fixing is then performed based on these candidates and fully relies on the eventually erroneous float ambiguity covariance matrix.

As the covariance matrix might be erroneous, we determine sets of integer candidates at multiple epochs using different float solutions to increase the likelihood of including the correct candidate in the set of all candidates.

The sets of candidate vectors are merged, i.e. redundant candidates are eliminated and all previous candidates are transformed in case of changing reference satellites. The obtained set of merged candidates are stacked in the pool

$$
N_{\text {pool }}=\left\{N_{12}^{(1)}, \ldots, N_{12}^{(c)}\right\}
$$

Note that the lengths of the candidate vectors might vary according to the number of tracked satellites at the selected epoch of the float solution. The consideration of the float solution at multiple epochs is of essential importance as the subsequent candidate selection can only be successful if the 
correct candidate is included in the set. The probability of correct fixing is written as

$$
P_{\text {cf }}=P_{\text {candidate in set }} \cdot P_{\text {correct candidate selected }} \text {. }
$$

\section{- Candidate tracking:}

The LAMBDA method [3] performs a fixing decision using the float solution at a certain epoch. The fixing is trusted if the ratio between the second-lowest and lowest sum of squared ambiguity residuals exceeds a certain value. We have analyzed numerous datasets and have observed that an erroneous candidate is often selected as both the ambiguity residuals and measurement residuals are not trustworthy. There is a strong need for enhancing the fixing decision. One option is to track the candidate vectors over time and to determine a phase-only least-squares baseline estimate at every epoch for every candidate, and to accumulate the sum of squared residuals over time, i.e.

$$
\sum_{j \in j_{s}}\left\|r_{\varphi_{12}}^{(m)}\left(t_{j}\right)\right\|^{2} \forall m
$$

with

$$
\begin{aligned}
r_{\varphi_{12}}^{(m)}\left(t_{j}\right) & =\lambda\left(\varphi_{12}\left(t_{j}\right)-\check{N}_{12}^{(m)}\right)-H\left(t_{j}\right) \check{\vec{b}}_{12}^{(m)}\left(t_{j}\right) \\
& =P_{H}^{\perp}\left(t_{j}\right) \lambda\left(\varphi_{12}\left(t_{j}\right)-\check{N}_{12}^{(m)}\right)
\end{aligned}
$$

with $P_{H}^{\perp}$ being the projector on the orthogonal complement of the space of $H$. The analysis of numerous datasets has shown a certain improvement but a reliable fixing can still not be guaranteed. Therefore, we additionally analyze the error of the least-squares baseline estimate for the $m$-th candidate at epoch $t_{j}$ :

$$
\Delta \check{\vec{b}}_{12}^{(m)}\left(t_{j}\right)=S\left(t_{j}\right) \lambda\left(\Delta \varphi_{\mathrm{MP}_{12}}\left(t_{j}\right)-\Delta \check{N}_{12}^{(m)}\right),
$$

with

$$
S\left(t_{j}\right)=\left(H^{\mathrm{T}}\left(t_{j}\right) \Sigma^{-1}\left(t_{j}\right) H\left(t_{j}\right)\right)^{-1} H^{\mathrm{T}}\left(t_{j}\right) \Sigma^{-1}\left(t_{j}\right),
$$

and $\Delta \check{N}_{12}^{(m)}$ being the error of the $m$-th integer candidate. The fixed phase residuals are shifted by

$$
\Delta r_{\varphi_{12}}^{(m)}\left(t_{j}\right)=P_{H}^{\perp}\left(t_{j}\right) \lambda\left(\Delta \varphi_{\mathrm{MP}_{12}}\left(t_{j}\right)-\Delta \check{N}_{12}^{(m)}\right)
$$

As the $H$ matrix is continuously changing over time due to the satellite movement, erroneous candidates can be detected from the baseline drift or residual drift. The baseline drift is obtained from Eq. (21) as

$$
\begin{aligned}
\check{\vec{b}}_{12}^{(m)}\left(t_{j}\right)-\check{\vec{b}}_{12}^{(m)}\left(t_{1}\right) \\
=S\left(t_{j}\right)\left(\lambda\left(\Delta \varphi_{\mathrm{MP}_{12}}\left(t_{j}\right)-\Delta \check{N}_{12}^{(m)}\right)+\varepsilon_{12}\left(t_{j}\right)\right) \\
\quad-S\left(t_{1}\right)\left(\lambda\left(\Delta \varphi_{\mathrm{MP}_{12}}\left(t_{1}\right)-\Delta \check{N}_{12}^{(m)}\right)+\varepsilon_{12}\left(t_{1}\right)\right) \\
=S\left(t_{j}\right) \lambda \Delta \varphi_{\mathrm{MP}_{12}}\left(t_{j}\right)-S\left(t_{1}\right) \lambda \Delta \varphi_{\mathrm{MP}_{12}}\left(t_{1}\right) \\
\quad+\left(S\left(t_{j}\right)-S\left(t_{1}\right)\right) \Delta \check{N}_{12}^{(m)} \\
\quad+S\left(t_{j}\right) \varepsilon_{12}\left(t_{j}\right)-S\left(t_{1}\right) \varepsilon_{12}\left(t_{1}\right) .
\end{aligned}
$$

Unfortunately, the drift due to the change of $H$ is rather small and compensated to some extent by the change of the weighting $\Sigma^{-1}$ and by the change of the phase multipath over time. However, the drift due to an erroneous integer candidate continuously grows over time. Therefore, the drift is estimated from the baseline estimates of numerous epochs, which are modeled as a linear function of time, i.e.

$$
\begin{gathered}
\left(\begin{array}{c}
\check{\vec{b}}_{12}^{(m)}\left(t_{1}\right)-\check{\vec{b}}_{12}^{(m)}\left(t_{1}\right) \\
\check{\vec{b}}_{12}^{(m)}\left(t_{2}\right)-\check{\vec{b}}_{12}^{(m)}\left(t_{1}\right) \\
\vdots \\
\check{\vec{b}}_{12}^{(m)}\left(t_{j}\right)-\check{\vec{b}}_{12}^{(m)}\left(t_{1}\right)
\end{array}\right) \\
=\Lambda\left(\begin{array}{c}
\alpha_{0}^{(m)} \\
\alpha_{1}^{(m)}
\end{array}\right)+\left(\begin{array}{c}
\eta_{12}^{(m)}\left(t_{1}\right) \\
\eta_{12}^{(m)}\left(t_{2}\right) \\
\vdots \\
\eta_{12}^{(m)}\left(t_{j}\right)
\end{array}\right),
\end{gathered}
$$

with $\alpha_{0}^{(m)}$ and $\alpha_{1}^{(m)}$ being the linear coefficients and $\eta_{12}^{(m)}\left(t_{j}\right)$ including the difference of the projected phase noise and multipath, and

$$
\Lambda=\left(\begin{array}{cc}
1 & t_{1}-t_{1} \\
1 & t_{2}-t_{1} \\
\vdots & \vdots \\
1 & t_{j}-t_{1}
\end{array}\right)
$$

The coefficients are obtained by a standard least-squares estimation:

$$
\left(\begin{array}{c}
\hat{\alpha}_{0}^{(m)} \\
\hat{\alpha}_{1}^{(m)}
\end{array}\right)=\left(\Lambda^{\mathrm{T}} \Lambda\right)^{-1} \Lambda^{\mathrm{T}}\left(\begin{array}{c}
\check{\vec{b}}_{12}^{(m)}\left(t_{1}\right)-\check{\overrightarrow{\vec{b}}}_{12}^{(m)}\left(t_{1}\right) \\
\overrightarrow{\vec{b}}_{12}^{(m)}\left(t_{2}\right)-\check{\vec{b}}_{12}^{(m)}\left(t_{1}\right) \\
\vdots \\
\check{\vec{b}}_{12}^{(m)}\left(t_{j}\right)-\check{\vec{b}}_{12}^{(m)}\left(t_{1}\right)
\end{array}\right) .
$$

The coefficient $\alpha_{1}^{(m)}$ describes the slope/ drift of the baseline due to the erroneous candidate and to some extent due to phase multipath and noise.

- Candidate selection:

The LAMBDA method [3] selects the candidate solely based on the sum of squared ambiguity residuals. This makes the fixing very sensitive to any error in the float solution.

We perform a pre-selection of integer candidates based on the accumulated sum of fixed phase measurement residuals of Eq. (19), i.e. by selecting only the candidates where the accumulated sum of residuals is below a predefined threshold $\gamma$. The candidate with the maximum baseline stability is then chosen among all preselected candidates, i.e.

$$
\check{N}_{12}^{(m)}=\arg \min _{m}\left|\hat{\alpha}_{1}^{(m)}\right| \quad \text { s.t } \sum_{j \in j_{s}}\left\|r_{\varphi_{12}}^{(m)}\left(t_{j}\right)\right\|^{2} \stackrel{!}{<} \gamma .
$$

The joint consideration of the residuals and the baseline stability results in a much more reliable fixing.

An erroneous candidate might still be selected if the integer term in Eq. (24) compensates for the multipath and noise 
terms, i.e. an erroneous candidate has a lower drift than the correct candidate.

The change of the phase multipath over time is in general not as continuous as the change of the $H$ matrix over time. Thus, the presence of phase multipath could be detected by analyzing the residuals of the drift estimation, i.e.

$$
r_{\hat{\alpha}_{0}^{(m)}, \hat{\alpha}_{1}^{(m)}}=P_{\Lambda}^{\perp}\left(\begin{array}{c}
\check{\vec{b}}_{12}^{(m)}\left(t_{1}\right)-\check{\overrightarrow{\vec{b}}}_{12}^{(m)}\left(t_{1}\right) \\
\check{\vec{b}}_{12}^{(m)}\left(t_{2}\right)-\check{\vec{b}}_{12}^{(m)}\left(t_{1}\right) \\
\vdots \\
\check{\vec{b}}_{12}^{(m)}\left(t_{j}\right)-\check{\vec{b}}_{12}^{(m)}\left(t_{1}\right)
\end{array}\right),
$$

with $P_{\Lambda}^{\perp}$ being the projector on the orthogonal complement of the space of $\Lambda$. As the least-squares baseline estimates $\check{\vec{b}}_{12}^{(m)}\left(t_{j}\right)$ are dependent on $\Sigma\left(t_{j}\right)$ and any variation of $\Sigma\left(t_{j}\right)$ might lead to a variation of $\overrightarrow{\vec{b}}_{12}^{(m)}\left(t_{j}\right)$, it is recommended to use of constant $\Sigma$ during the candidate tracking and selection phases.

\section{Measurement results}

This section includes the verification of our RTK positioning with multipath estimation using real measurements.

As the initial carrier phase ambiguity resolution is the most challenging part and as reproducible conditions are needed for performance testing, we limit ourselves to static conditions. The baseline length between two single frequency receivers can be up to $10 \mathrm{~km}$ to suppress the ionospheric delay sufficiently by double differencing in nominal ionospheric conditions. We consider a baseline length of $104 \mathrm{~m}$ to enable data transmission via Bluetooth.

We mounted two ANavS GPS modules with u-blox LEA 6T GPS receivers and patch antennas on a grainfield near Aschheim, Germany, on July 20, 2015. The receivers of the LEA 6T series can track 50 channels and have a sensitivity of $-162 \mathrm{dBm}$ for tracking and navigation [10]. Open-sky conditions resulted in 8 to 10 visible GPS satellites with an elevation of at least 10 degrees. Artificial code multipath was added to the real code measurement of each satellite to test the performance of multipath estimation.

The code multipath was simulated as a random walk process:

$$
\Delta \rho_{\mathrm{MP}_{r, n}}^{k}=\Delta \rho_{\mathrm{MP}_{r, n-1}}^{k}+\eta_{\Delta \rho_{\mathrm{MP} r, n}^{k}} .
$$

The initial value of the code multipath at epoch $n=0$ was chosen as a random value using a normal distribution with a standard deviation of $3 \mathrm{~m}$. The change of the code multipath between two successive epochs was also set to a random value using a normal distribution with a standard deviation of 0.2 $\mathrm{m}$. The code multipath was determined for each satellite independently.

The float solution was obtained with a Kalman filter, in which the baseline coordinates, the DD ambiguities and the DD code multipath parameters were determined jointly. Fig. 1 shows the code multipath for each double difference over time. One can observe that the change of the code multipath between two successive epochs is for most epochs much lower than the absolute value of the code multipath. This high temporal correlation of the multipath is typical for static environments and is fully exploited in the Kalman filter. The enlarged sections show that the estimated code multipath closely follows the true multipath. The deviation between the estimated and true multipath is only in the order of magnitude of the code noise.

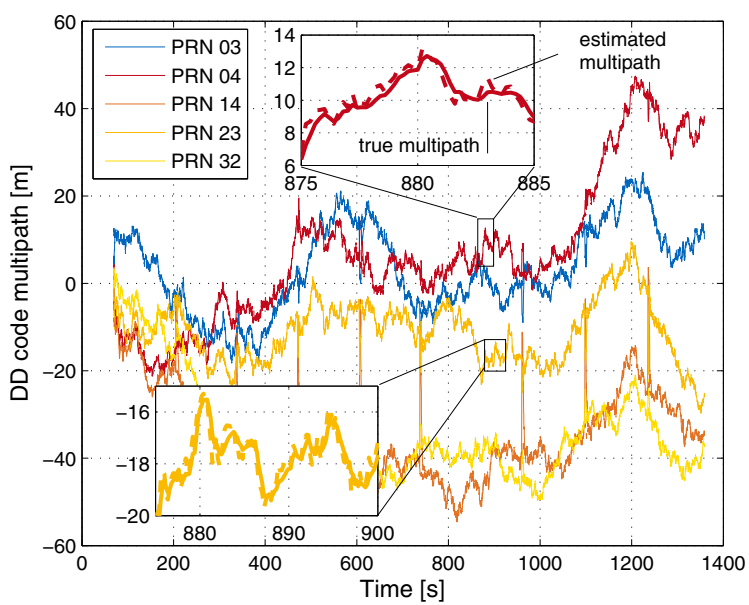

Figure 1. Estimation of DD code multipath: The true and estimated multipath are shown for all visible satellites. The enlarged sections for PRN 04 and 14 show that the estimated code multipath estimates closely follow the track of the true ones. The error of the multipath estimates are only in the order of magnitude of the code noise.

Fig. 2 shows the accuracy of the float baseline estimate. We can observe that the baseline error reduces to less than $0.5 \mathrm{~m}$ within a few seconds, and then varies by only a few decimeters. The sudden increases in uncertainty every $\sim 200 \mathrm{~s}$ are caused by restarts of the Kalman filter after each ambiguity fixing. If the code multipath is not estimated, the covariance matrix of the code noise has to be increased dramatically to absorb the code multipath in the code noise. The enlarged code noise covariance matrix would result in much longer convergence times.

The small error of the float solution shows that the estimation of the code multipath prevents a mapping of the code multipath into other state parameters. Consequently, the estimation of the code multipath is very beneficial.

Fig. 3 shows the DD phase residuals for the fixed solution over time. The residuals of all DD are less than $2 \mathrm{~cm}$ and almost drift-free, which indicates a consistent measurement model and a correct integer ambiguity fixing and cycle slip correction. The slight long-term variations are caused by phase multipath, by a changing satellite geometry and/ or by a change of the weighting of the measurements.

Fig. 4 shows the error of the least-squares estimate of the baseline coordinates using only the carrier phase measurements after ambiguity fixing. A millimetre-level positioning accuracy is achievable with low-cost GPS receivers and patch antennas despite severe code multipath. The interpretation is very similar to the previous figure, i.e. the small order of magnitude of only $1 \mathrm{~cm}$ indicates a consistent measurement model and a correct ambiguity fixing. The slight longterm variations are due to phase multipath, by a changing satellite geometry and/ or by a change of the weighting of the measurements. 


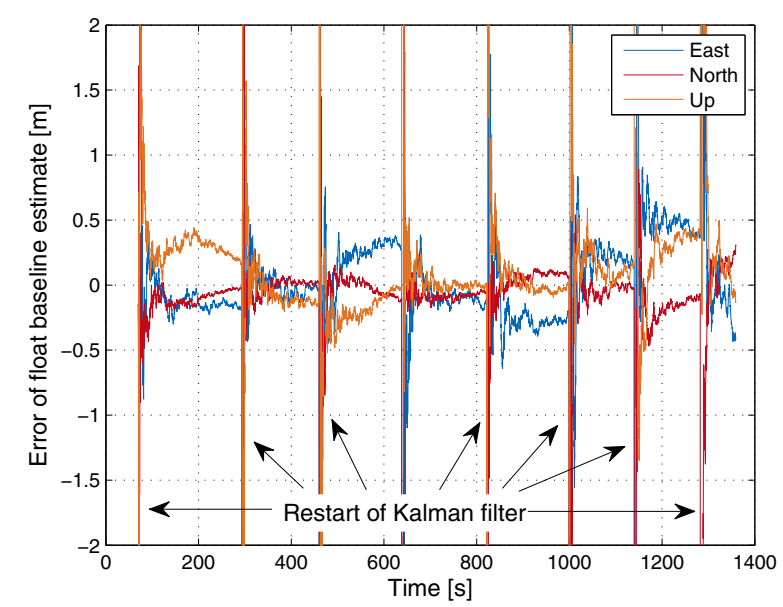

Figure 2. Accuracy of Kalman filter based float solution with baseline, ambiguity and code multipath estimation: The baseline errors reduce to less than $0.5 \mathrm{~m}$ after convergence of the Kalman filter. This error is in the order of the code noise and much lower than the code multipath. The sudden increases in uncertainty every $\sim 200 \mathrm{~s}$ arise from restarts of the filter after integer ambiguity fixing.

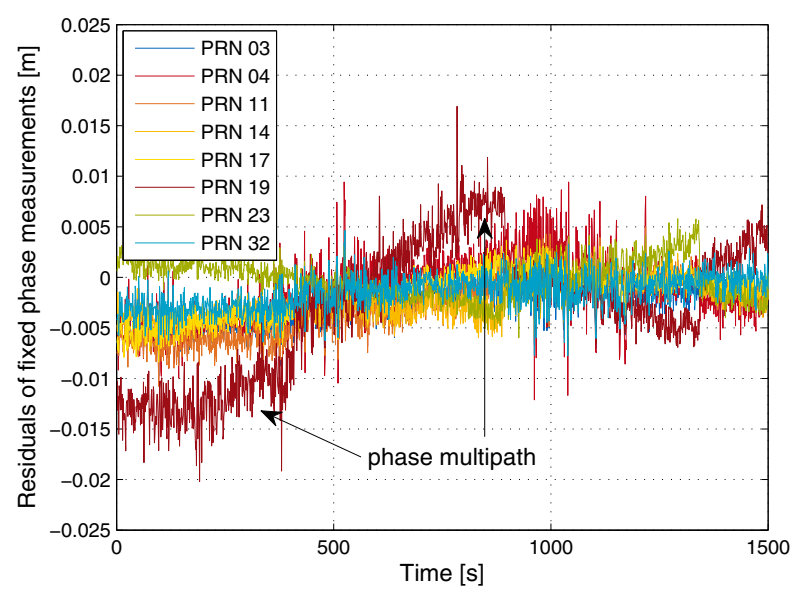

Figure 3. Residuals of fixed DD carrier phase measurements: The residuals are less than $2 \mathrm{~cm}$, which indicates a correct ambiguity fixing and correct cycle slip correction. The slight long-term variations are caused by phase multipath, by a changing satellite geometry and/ or by a change of the adaptive weighting of the measurements.

\section{CONCLUSION}

Multipath is the challenge for RTK positioning with low-cost GPS receivers and antennas. In this paper, we exploited the temporal correlation of the code multipath to enhance the RTK performance. We estimate a code multipath parameter for each double difference in the float solution. The multipath is modeled as a scaled Gauss-Markov process to cover both static and kinematic multipath. The ambiguity fixing includes two phases: an integer collection phase and an integer tracking phase. In the collection phase, we determine candidate vectors at multiple epochs to increase the likelihood of including the correct candidate vector in the set of candidate vectors. In the tracking phase, we determine a

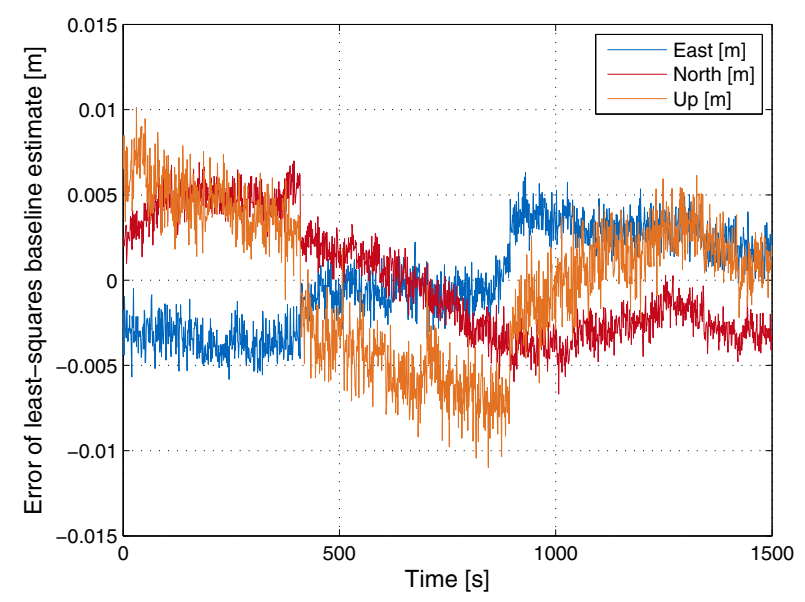

Figure 4. Error of least-squares ambiguity fixed baseline estimate: A millimetre-level positioning accuracy is achieved with low-cost GPS receivers despite severe code multipath of up to $50 \mathrm{~m}$. The slight temporal variations are caused by phase multipath, by a changing satellite geometry and/ or by a change of the weighting of the measurements.

single epoch phase-only least-squares fixed baseline estimate for each candidate at each epoch. The residuals are accumulated over time and the candidate vector is selected based on the minimum baseline drift to enhance the reliability of the candidate selection. We applied the proposed method to real measurements of two low-cost GPS receivers and patch antennas, and obtained a millimetre-level positioning accuracy despite code multipath of up to $50 \mathrm{~m}$.

\section{REFERENCES}

[1] Herbert Landau, Ulrich Vollath and Xiaoming Chen, Virtual Reference Station Systems, Journal of Global Positioning Systems (CGPS), pp. 137-143, 2002.

[2] Ulrich Vollath, Alois Bücherl, Herbert Landau, Christian Pagels and Bernhard Wagner, Multi-Base RTK Positioning using Virtual Reference Stations, Proc. of ION, 2000.

[3] Peter J.G. Teunissen, The least-squares ambiguity decorrelation adjustment: a method for fast GPS integer ambiguity estimation, Journal of Geodesy, pp. 65-82, vol. 70, 1995.

[4] Robert G. Brown and Patrick Y.C. Hwang, Introduction to Random Signals and Applied Kalman Filtering with Matlab Exercises, 4th edition, Wiley, 2012.

[5] Patrick S. Henkel and Michele Iafrancesco, Tightly coupled Position and Attitude Determination with two low-cost GNSS receivers, Proc. of 11-th Intern. IEEE Symp. on Wireless Communication Systems (ISWCS), Barcelona, Spain, pp. 895-900, Aug. 2014.

[6] Patrick S. Henkel, Tightly coupled Precise Point Positioning and Attitude Determination, IEEE Transactions on Aerospace and Electronic Systems, accepted, 2015.

[7] Juan M. Cárdenas, Precise attitude determination with low-cost GNSS receivers, Master thesis, Technische Universität München, Mar. 2013.

[8] Andreas Sperl, Joint RTK and Attitude determination, Master thesis, Technische Universität München, Sep. 2015. 
[9] Michele Iafrancesco, GPS/ INS tightly coupled position and attitude determination with low-cost sensors, Master thesis, Technische Universität München, Sep. 2015.

[10] u-blox, LEA-6 GPS modules data sheet, https://www.ublox.com/sites/default/files/products/documents/LEA6_DataSheet_(GPS.G6-HW-09004).pdf, accessed online: Jan. 11, 2016.

\section{BIOGRAPHY}

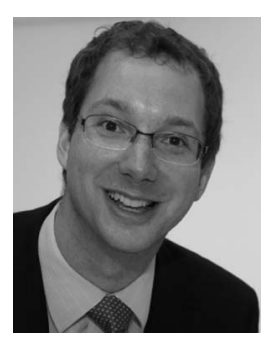

Patrick Henkel has received his Bachelor, Master and PhD degrees (summa cum laude) from the Technische Universität München, Germany. He is currently working on his habilitation on Precise Point Positioning at TUM. Dr. Henkel received the Pierre Contensou Gold Medal in 2007, the 1st prize in Bavaria at the European Satellite Navigation Competition in 2010 and a dissertation award of the Vodafone Research Foundation for his PhD thesis in 2011. He founded ANavS in 2011 and is currently also the managing director.

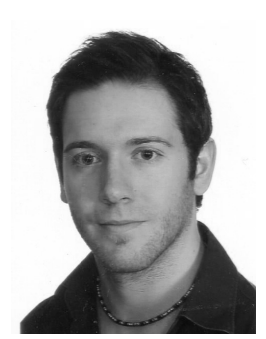

Andreas Sperl has served an apprenticeship to be an Electronics Technician for Electronic devices and systems. He additionally studied Electrical and Computer Engineering at the Technische Hochschule Deggendorf and the Technische Universität München. Mr. Sperl wrote his master thesis on "Joint RTK and Attitude determination" at ANavS. He is currently working on RTK positioning as a software and hardware developer at ANavS. 